\title{
CAUCHY INTEGRAL EQUALITIES AND APPLICATIONS
}

\author{
BOO RIM CHOE
}

\begin{abstract}
We study bounded holomorphic functions $\pi$ on the unit ball $B_{n}$ of $\mathbb{C}^{n}$ satisfying the following so-called Cauchy integral equalities:

$$
C\left[\pi^{m+1} \bar{\pi}\right]=\gamma_{m} \pi^{m} \quad(m=0,1,2, \ldots)
$$

for some sequence $\gamma_{m}$ depending on $\pi$. Among the applications are the AhernRudin problem concerning the composition property of holomorphic functions on $B_{n}$, a projection theorem about the orthogonal projection of $H^{2}\left(B_{n}\right)$ onto the closed subspace generated by holomorphic polynomials in $\pi$, and some new information about the inner functions. In particular, it is shown that if we interpret $\operatorname{BMOA}\left(B_{n}\right)$ as the dual of $H^{1}\left(B_{n}\right)$, then the map $g \rightarrow g \circ \pi$ is a linear isometry of $\operatorname{BMOA}\left(B_{1}\right)$ into $\operatorname{BMOA}\left(B_{n}\right)$ for every inner function $\pi$ on $B_{n}$ such that $\pi(0)=0$.
\end{abstract}

\section{INTRODUCTION}

Let $n$ be a positive integer. Throughout the paper $\mathbb{C}^{n}$ denotes the $n$ dimensional complex space with corresponding unit ball $B=B_{n}$ whose boundary is the unit sphere $S=S_{n}$. The unique normalized rotation-invariant Borel measure on $S$ is denoted by $\sigma=\sigma_{n}$. When $n=1$, we use more customary notation $U$ and $T$ in place of $B_{1}$ and $S_{1}$, respectively. The symbol $P_{n}$ stands for the class of holomorphic homogeneous polynomials $\pi$ on $\mathbb{C}^{n}$ normalized so that $\pi(B)=U$. It is assumed that $n \geq 2$ unless the contrary is explicitly stated.

In their recent paper P. Ahern and W. Rudin have posed the following problem [AR 1, Problem 3.5]:

If $\pi$ is a function holomorphic on $B$ and continuous on $\bar{B}$ such that $\pi(B)=U$, then what additional hypotheses are needed to ensure that $\pi$ has the pullback property from $\mathscr{B}(U)$ to $\operatorname{BMOA}(B)$ (i.e., $g \circ \pi \in \operatorname{BMOA}(B)$ for every $g \in \mathscr{B}(U))$ ?

See $\S 2$ for notation. This Ahern-Rudin problem has not only its own interest but also some other applications. Its origin apparently goes back to $P$. Ahern's paper [A]. In [A] P. Ahern proved that $\pi(z)=n^{n / 2} z_{1} \cdots z_{n}$ has the pullback property

Received by the editors March 29, 1988.

1980 Mathematics Subject Classification (1985 Revision). Primary 32A35.

Key words and phrases. Cauchy Integral Equalities, the Ahern-Rudin problem, projection.

This work represents a part of the author's Ph.D. thesis completed under the supervision of Walter Rudin. 
from $\mathscr{B}(U)$ to $\operatorname{BMOA}(B)$ to derive the pathological boundary behavior near the maximum modulus set $\operatorname{Max} \pi=\pi^{-1}(T) \cap S$ of some BMOA-functions on $B$. His method was used by P. Russo [Rus] to prove the same property of the sum-of-squares $\pi(z)=z_{1}^{2}+\cdots+z_{n}^{2}$ for the same purpose (D. Ullrich [U] has recently obtained a penetrating result concerning the boundary behavior of BMOA-functions). Then P. Ahern and W. Rudin [AR1] found a new proof of these results which allowed them to obtain the same property of every monomial $\pi \in P_{n}$ and observed new consequences concerning the boundary zeros of functions holomorphic on $B$. In [AR2] they derived another consequence, namely, Paley-type gap theorems which have no analogue in the one variable case.

The main observation in [AR1] leading to a new proof of the above BMOAresults in [A] and [Rus] is that if $\pi \in P_{n}$ is a monomial or the sum-of-squares, then the sequence $C\left[\pi^{m+1} \bar{\pi}\right]$ of Cauchy integrals satisfies the following:

$$
C\left[\pi^{m+1} \bar{\pi}\right]=\gamma_{m} \pi^{m} \quad(m=0,1,2, \ldots)
$$

for some sequence $\gamma_{m}$ satisfying

$$
\gamma_{m}=1-\frac{\delta(\pi)}{2 m}+O\left(\frac{1}{m^{2}}\right) \text { as } m \rightarrow \infty
$$

where $\delta(\pi)>0$ is the topological co-dimension of Max $\pi$ in $S$. More precisely, $\delta(\pi)=(2 n-1)-\operatorname{dim}(\operatorname{Max} \pi)$ where $\operatorname{dim}(\operatorname{Max} \pi)$ denotes the topological dimension of $\operatorname{Max} \pi$. For the positivity of $\delta(\pi)$, see [Ru2, $\S 11.4]$.

For brevity this sequence of equalities will be called the Cauchy Integral Equalities (CIE). Note that if CIE holds as above, then it is necessary that

$$
\gamma_{m}=\int_{S}|\pi|^{2 m+2} d \sigma / \int_{S}|\pi|^{2 m} d \sigma
$$

and therefore $\gamma_{m} \rightarrow\|\pi\|_{\infty}^{2}=1$ as $m \rightarrow \infty$ if $\pi \in P_{n}$.

In $\S 2$ we briefly describe standard function spaces and related facts that occur in this paper. In $\S 3$ it is shown that every $\pi$ belonging to a certain subclass of $P_{n}$ satisfies CIE with the same estimate $(*)$ and therefore solves the Ahern-Rudin problem (see Theorem 3.7). This will be based on two previous cases: roughly speaking, such $\pi$ is a direct product of powers of two special kinds of functions mentioned above. But the example given at the end of the section shows that CIE holds only for very special $\pi \in P_{n}$ and therefore suggests that something analogous to Theorem 3.7 is as far as we can go by using CIE method. This is why we study in $\S 4$ some general facts, Theorems 4.5 and 4.6 , related to the Ahern-Rudin problem which reveal a close connection between this problem and the orthogonal projection $Q_{\pi}$ of the Hardy space $H^{2}(B)$ onto $X^{2}(\pi)$, the closure in $H^{2}(B)$ of holomorphic polynomials in $\pi$. If one wishes to attack the Ahern-Rudin problem for the whole class $P_{n}$, Theorem 4.5 naturally yields two questions which may be of some independent interest (see Definition 4.1 for notation): 
Question A. If $\pi \in P_{n}$, then does $Q_{\pi}$ extend to a bounded projection of $H^{1}(B)$ onto $X^{1}(\pi)$ ?

Question B. If $\pi \in P_{n}$, then does $A^{1}(\pi)^{*}=\mathscr{B}(U)$ hold?

The answer to Question A is yes if $\pi$ satisfies CIE: Theorem 5.3 contains a much stronger result for such $\pi$. Note that $Q_{\pi}$ extends to an integral operator (see Example 4.2(2)) of $L^{1}(\sigma)$ into the space of holomorphic functions on $B$. In this context the situation is quite different: it follows from [K, Theorem (10)] that $Q_{\pi}$ is always unbounded on $L^{1}(\sigma)$. The answer to Question B is also yes if $\pi$ belongs to a large subclass (called $\Omega_{n}$ in [C2]) of $P_{n}$ containing all functions $\pi$ as in Theorem 3.7. One can deduce this fact from the main result of [C2]: the details can be found in [C1]. Also note that these observations provide another proof of Theorem 3.7.

Finally in $\S 5$ we derive another consequence of CIE concerning the orthogonal projections $Q_{\pi}$ (see Theorem 5.3). Such a projection theorem was previously proved by W. Rudin [Ru1, Theorem 4.5] in the special case when $\pi$ is an inner function such that $\pi(0)=0$. This projection theorem and Theorems 4.5 and 4.6 imply an interesting consequence, Theorem 5.4:

if $\pi$ is an inner function on $B(n \geq 1)$ such that $\pi(0)=0$, then the map $g \rightarrow g \circ \pi$ is a linear isometry of $\operatorname{BMOA}(U)$ into $\operatorname{BMOA}(B)$.

Note that a similar (and easy) result in the $H^{p}$-context also holds.

\section{Preliminaries}

Throughout this section $n \geq 1$. Let $1 \leq p<\infty$. The Hardy space $H^{p}(B)$ consists of functions $f$ holomorphic on $B$ such that

$$
\|f\|_{H^{p}(B)}^{p}=\sup _{0<r<1} \int_{S}|f(r \xi)|^{p} d \sigma(\xi)<\infty .
$$

It is well known (see [Ru2, Theorem 5.6.4]) that if $f \in H^{p}(B)$, then $f^{*}(\xi)=$ $\lim _{r \rightarrow 1_{-}} f(r \xi)$ exists for $[\sigma]$ a.e. $\xi \in S$. Moreover, the map $f \rightarrow f^{*}$ is a linear isometry of $H^{p}(B)$ onto $H^{p}(S)$, the closed subspace of $L^{p}(\sigma)$ consisting of all boundary values of $H^{p}$-functions (see [Ru2, Theorem 5.6.8]). This is the reason why we often use the same letter for a function $f \in H^{p}(B)$ and its boundary function $f \in L^{p}(\sigma)$. For simplicity we will also identify $H^{p}(B)$ with $H^{p}(S)$.

The Cauchy integral of a function $f \in L^{1}(\sigma)$ will be denoted by $C[f]$. We shall use the fact that the map $f \rightarrow C[f]$, when restricted to $L^{2}(\sigma)$, is the orthogonal projection of $L^{2}(\sigma)$ onto $H^{2}(B)$. For details see [Ru2, Sections 3.2 and 5.6].

The most convenient definition of $\operatorname{BMOA}(B)$ for our purpose is perhaps a little bit artificial: $\operatorname{BMOA}(B)$ consists of all $f=C[\psi]$ where $\psi \in L^{\infty}(\sigma)$. The BMOA-norm of $f$ is

$$
\|f\|_{\mathrm{BMOA}(B)}=\inf \left\{\|\psi\|_{L^{\infty}(\sigma)}: C[\psi]=f\right\} .
$$


It is easily seen via the Hahn-Banach theorem that $\operatorname{BMOA}(B)$ is isometrically isomorphic to the dual of $H^{1}(B)$. More explicitly, given a bounded linear functional $\Lambda$ on $H^{1}(B)$, there exists a unique $f \in \operatorname{BMOA}(B)$ such that

$$
\Lambda(g)=\int_{S} g \bar{f} d \sigma \quad \text { for every } g \in H^{2}(B) .
$$

In addition, if this is the case, then $\|\Lambda\|=\|f\|_{\mathrm{BMOA}(B)}$. It is clear from this definition that $H^{\infty}(B) \subset \operatorname{BMOA}(B) \subset H^{p}(B)$ for all $p<\infty$. The usual definition of $\operatorname{BMOA}(B)$ is as follows: $\operatorname{BMOA}(B)$ consists of all $f \in H^{2}(B)$ whose boundary functions $f^{*}$ are functions of bounded mean oscillations with respect to the nonisotropic metric on $S$ that corresponds to the Koranyi approach regions. It then turns out that $\operatorname{BMOA}(B)$ has a natural identification with the dual of $H^{1}(B)$ : see [G] for one variable and [CRW] for several variables.

The Bloch space $\mathscr{B}(U)$ is the space of all functions $f$ holomorphic on $U$ such that

$$
\sup _{\lambda \in U}\left(1-|\lambda|^{2}\right)\left|f^{\prime}(\lambda)\right|<\infty .
$$

In the present paper we will not use any general property of Bloch functions and refer to [G] and [ACP] for a more detailed description of the Bloch space.

The class of nonconstant bounded holomorphic functions $\pi$ on $B$ such that $\sup _{z \in B}|\pi(z)|=1$ will be denoted by $H_{n}$. Finally the notation $\langle$,$\rangle denotes$ the inner product on $L^{2}(\sigma)$. The dimension involved in $C[f]$ or $\langle$,$\rangle will be$ clear from the context.

\section{CAUChY INTEGRAL EQUALITIES}

We shall show that CIE holds for a certain, but very special, class of functions $\pi \in P_{n}$ with the same estimate $(*)$. The so-called integration by separation will play an important role in generalization of known cases. As immediate consequences we will observe the gap theorems and the pathological boundary behavior of some BMOA-functions.

Before going further we introduce some notation. Suppose $\psi$ and $\phi$ are $\mathbb{C}$-valued functions on $E \subset \mathbb{C}^{k}$ and $F \subset \mathbb{C}^{l}$, respectively. We shall let $\psi \otimes \phi$ denote the function on $E \times F \subset \mathbb{C}^{k} \times \mathbb{C}^{l}=\mathbb{C}^{k+l}$ defined by $(\psi \otimes \phi)(z, w)=$ $\psi(z) \phi(w)$ for $(z, w) \in E \times F$. For more than two functions $\phi_{1}, \ldots, \phi_{p}$, $\bigotimes_{i=1}^{p} \phi_{i}$ is defined inductively.

The following integral formula has been proved in [C2]. The symbol $\nu_{k}$ denotes the normalized volume measure on $\mathbb{C}^{k}$.

Lemma 3.1 (Integration by averaging). If $k$ and $l$ are positive integers such that $n=k+l$, then for every $f \in L^{1}\left(\sigma_{n}\right)$

$$
\int_{S_{n}} f d \sigma_{n}=\left(\begin{array}{c}
n-1 \\
k
\end{array}\right) \int_{B_{k}} \int_{S_{l}} f\left(z, \sqrt{1-|z|^{2}} \xi\right) d \sigma_{l}(\xi)\left(1-|z|^{2}\right)^{l-1} d \nu_{k}(z)
$$

holds. 
In the following a continuous function $f$ on $\mathbb{C}^{n} \backslash 0$ is called positively homogeneous of degree $d$ if

$$
f(t z)=t^{d} f(z) \quad\left(t>0, z \in \mathbb{C}^{n} \backslash 0\right) .
$$

Proposition 3.2. Suppose that $k$ and $l$ are positive integers such that $n=k+l$. Let $d>-l$ and $\phi$ be a positively homogeneous function on $\mathbb{C}^{n} \backslash 0$ of degree $2 d$ such that $\int_{S_{l}} \phi d \sigma_{l}=1$. Then

$$
\int_{S_{n}} \psi \otimes \phi d \sigma_{n}=\left(\begin{array}{c}
n-1 \\
k
\end{array}\right) \int_{B_{k}} \psi(z)\left(1-|z|^{2}\right)^{l+d-1} d \nu_{k}(z)
$$

for every $\psi \in L^{1}(\tau)$ where $d \tau(z)=\left(1-|z|^{2}\right)^{l+d-1} d \nu_{k}(z)$ on $B_{k}$.

Proof. This is immediate from Lemma 3.1 and homogeneity of $\phi$.

Proposition 3.3 (Integration by separation). Let $\psi$ and $\phi$ be positively homogeneous functions on $\mathbb{C}^{k} \backslash 0$ and $\mathbb{C}^{l} \backslash 0$, respectively. Suppose that $\psi$ and $\phi$ are of degree $2 s$ and $2 d$, respectively, where $s>-k$ and $d>-l$. Let $n=k+l$. Then

$$
\int_{S_{n}} \psi \otimes \phi d \sigma_{n}=\frac{\Gamma(n) \Gamma(k+s) \Gamma(l+d)}{\Gamma(n+d+s) \Gamma(k) \Gamma(l)} \int_{S_{k}} \psi d \sigma_{k} \int_{S_{l}} \phi d \sigma_{l} .
$$

Proof. We may assume that $\int_{S_{k}} \psi d \sigma_{k}=1=\int_{S_{l}} \phi d \sigma_{l}$. By Proposition 3.2,

$$
\int_{S_{n}} \psi \otimes \phi d \sigma_{n}=\left(\begin{array}{c}
n-1 \\
k
\end{array}\right) \int_{B_{k}} \psi(z)\left(1-|z|^{2}\right)^{l+d-1} d \nu_{k}(z) .
$$

Compute the right side of the above in polar coordinates by using homogeneity and obtain $\Gamma(n) \Gamma(k+s) \Gamma(l+d) / \Gamma(n+d+s) \Gamma(k) \Gamma(l)$.

By repeating integration by separation one obtains the following corollary. Note that the familiar formula of the $L^{2}(\sigma)$-norm of holomorphic monomials on $\mathbb{C}^{n}$ is a special case of this corollary.

Corollary 3.4. Let $\phi_{i}$ be positively homogeneous functions on $\mathbb{C}^{l_{i}} \backslash 0$ of degree $2 d_{i}$ where $d_{i}>-l_{i}$ for $i=1, \ldots, p$. Let $n=l_{1}+\cdots+l_{p}$ and $d=d_{1}+\cdots+d_{p}$. Then

$$
\int_{S_{n}} \bigotimes_{i=1}^{p} \phi_{i} d \sigma_{n}=\frac{\Gamma(n)}{\Gamma(n+d)} \prod_{i=1}^{p} \frac{\Gamma\left(l_{i}+d_{i}\right)}{\Gamma\left(l_{i}\right)} \int_{S_{l_{i}}} \phi_{i} d \sigma_{l_{i}} .
$$

In the following lemma $j$ denotes the constant function 1 on $\mathbb{C}^{l}$.

Lemma 3.5. Let $\psi \in P_{k}$. Suppose $\phi \in P_{l}$ or $\phi=j$. Define $\pi=b \psi \otimes \phi$ where $b>0$ is chosen so that $\pi \in P_{n} \quad(n=k+l)$. If $\psi$ and $\phi$ satisfy CIE, then so does $\pi$. Moreover, for every $m$,

$$
\frac{\left\langle\pi^{m+1}, \pi^{m+1}\right\rangle}{\left\langle\pi^{m}, \pi^{m}\right\rangle}= \begin{cases}{\left[1-\frac{l}{m}+O\left(\frac{1}{m^{2}}\right)\right] a_{m}} & \text { if } \phi=j, \\ {\left[1-\frac{1}{2 m}+O\left(\frac{1}{m^{2}}\right)\right] a_{m} b_{m}} & \text { if } \phi \in P_{l},\end{cases}
$$


where

$$
a_{m}=\frac{\left\langle\psi^{m+1}, \psi^{m+1}\right\rangle}{\left\langle\psi^{m}, \psi^{m}\right\rangle} \text { and } b_{m}=\frac{\left\langle\phi^{m+1}, \phi^{m+1}\right\rangle}{\left\langle\phi^{m}, \phi^{m}\right\rangle} .
$$

Note. When $\operatorname{Max} \psi$ and $\operatorname{Max} \phi$ are manifolds, it is easily verified that if $\psi$ and $\phi$ satisfy $(*)$, then so does $\pi$.

Proof. Let $d=\operatorname{deg} \psi$ and $s=\operatorname{deg} \phi$. To show that $\pi$ satisfies $\mathrm{CIE}$, it is enough to show that there is a sequence $\gamma_{m}$ such that

$$
\left\langle\pi^{m+1}, \pi h\right\rangle=\gamma_{m}\left\langle\pi^{m}, h\right\rangle \quad(m=0,1,2, \ldots)
$$

for every monomial $h=f \otimes g$ where $f \in P_{k}$ and $g \in P_{l}$. Fix $m \geq 0$ and such an $h$. Note that both sides in (2) vanish unless $\operatorname{deg} f=d m$ and $\operatorname{deg} g=s m$. Thus we may assume that $\operatorname{deg} f=d m$ and $\operatorname{deg} g=s m$. It follows from the integration by separation that

$$
\begin{aligned}
\left\langle\pi^{m+1}, \pi h\right\rangle & =b^{m+2}\left\langle\psi^{m+1} \otimes \phi^{m+1}, \psi f \otimes \phi g\right\rangle \\
& =b^{m+2} \frac{\Gamma(n) \Gamma(k+m d+d) \Gamma(l+m s+s)}{\Gamma(k) \Gamma(l) \Gamma(n+m d+m s+d+s)} a_{m} b_{m}\left\langle\psi^{m}, f\right\rangle\left\langle\phi^{m}, g\right\rangle .
\end{aligned}
$$

On the other hand we have in a similar way

$$
\begin{aligned}
\left\langle\pi^{m}, h\right\rangle & =b^{m}\left\langle\psi^{m} \otimes \phi^{m}, f \otimes g\right\rangle \\
& =b^{m} \frac{\Gamma(n) \Gamma(k+m d) \Gamma(l+m s)}{\Gamma(k) \Gamma(l) \Gamma(n+m d+m s)}\left\langle\psi^{m}, f\right\rangle\left\langle\phi^{m}, g\right\rangle .
\end{aligned}
$$

Comparison of these two identities leads to (2) with

$$
\gamma_{m}=b^{2} a_{m} b_{m} \frac{\Gamma(k+m d+d) \Gamma(l+m s+s) \Gamma(n+m d+m s)}{\Gamma(k+m d) \Gamma(l+m s) \Gamma(n+m d+m s+d+s)} .
$$

Consider the case $\phi \in P_{l}$. Since $s \geq 1$, we have by (3)

(4) $\gamma_{m}=b^{2} a_{m} b_{m}\left(\prod_{i=0}^{d-1}(k+m d+i) \prod_{i=0}^{s-1}(l+m s+i)\right) / \prod_{i=0}^{d+s-1}(n+m d+m s+i)$.

Since $\gamma_{m} \rightarrow 1$ as $m \rightarrow \infty$, we obtain

$$
b^{2}=\frac{(d+s)^{d+s}}{d^{d} s^{s}} .
$$

Insert the above into (4), divide numerator and denominator by the constant that makes both monic and compute the coefficients of $m^{d+s-1}$. This yields (1) when $\phi \in P_{l}$.

If $\phi=j$, then $b=1, b_{m} \equiv 1$, and $s=0$. Thus from (3),

$$
\gamma_{m}=a_{m} \prod_{i=0}^{d-1}(k+m d+i) / \prod_{i=0}^{d-1}(n+m d+i) .
$$

This implies (1) when $\phi=j$. The proof is complete. 
Lemma 3.6. Suppose that $\pi \in P_{n}$ satisfies CIE. Then

(1) $\pi^{d}$ satisfies CIE for every positive integer $d$. In addition, if $\pi$ satisfies (*), then so does $\pi^{d}$.

(2) $\pi \circ \mathscr{U}$ satisfies CIE for every unitary transformation $\mathscr{U}$ on $\mathbb{C}^{n}$.

Proof. Let $\gamma_{m}=\left\langle\pi^{m+1}, \pi^{m+1}\right\rangle /\left\langle\pi^{m}, \pi^{m}\right\rangle$. Then we have by assumption

$$
C\left[\pi^{m+1} \bar{\pi}\right]=\gamma_{m} \pi^{m} \quad(m=0,1,2, \ldots) .
$$

Fix an arbitrary $h \in H^{2}(B)$. Then, for positive integers $k$ and $l$, we have

$$
\left\langle\pi^{l}, \pi^{k} h\right\rangle=\left\langle\pi^{l} \bar{\pi}, \pi^{k-1} h\right\rangle=\gamma_{l-1}\left\langle\pi^{l-1}, \pi^{k-1} h\right\rangle .
$$

Repeating this, we find for $l \geq k \geq 1$

$$
\left\langle\pi^{l}, \pi^{k} h\right\rangle=\gamma_{l-1} \cdots \gamma_{l-k}\left\langle\pi^{l-k}, h\right\rangle \text {. }
$$

In other words, $C\left[\pi^{l} \bar{\pi}^{k}\right]=\gamma_{l-1} \cdots \gamma_{l-k} \pi^{l-k}$. Thus

$$
C\left[\pi^{d(m+1)} \bar{\pi}^{d}\right]=\gamma_{d m+d-1} \cdots \gamma_{d m} \pi^{d m}
$$

and therefore $\pi^{d}$ satisfies CIE. Clearly $\delta(\pi)=\delta\left(\pi^{d}\right)$. Now if

$$
\gamma_{m}=1-\frac{\delta(\pi)}{2 m}+O\left(\frac{1}{m^{2}}\right) \text { as } m \rightarrow \infty
$$

then

$$
\gamma_{d m+i}=1-\frac{\delta(\pi)}{2(d m+i)}+O\left(\frac{1}{m^{2}}\right) \quad \text { as } m \rightarrow \infty,
$$

uniformly in $0 \leq i \leq d-1$. Also,

$$
\frac{1}{d m+i}=\frac{1}{d m}+O\left(\frac{1}{m^{2}}\right) \quad \text { as } m \rightarrow \infty,
$$

uniformly in $0 \leq i \leq d-1$. It follows that

$$
\gamma_{d m+d-1} \cdots \gamma_{d m}=1-\frac{\delta(\pi)}{2 m}+O\left(\frac{1}{m^{2}}\right) \text { as } m \rightarrow \infty \text {. }
$$

This proves (1). (2) is a consequence of the fact that Cauchy transform commutes with the action of unitary group [Ru2, Lemma 3.2.2]. The proof is complete.

We now come to the main result of this section which generalizes the results of P. Ahern, P. Russo, and Ahern-Rudin mentioned in $\S 1$.

Theorem 3.7. Let $\phi_{i} \in P_{l_{i}}$ be a monomial or a power of the sum-of-squares on $\mathbb{C}^{l_{i}}$ for $i=1, \ldots, p$. Define $\pi=b\left(\otimes_{i=1}^{p} \phi_{i}\right)$ where $b>0$ is chosen so that $\pi \in P_{n}\left(n=l_{1}+\cdots+l_{p}\right)$. Then $\pi$ has the pullback property from $\mathscr{B}(U)$ to $\operatorname{BMOA}(B)$.

Proof. By Lemma 3.6 each $\phi_{i}$ satisfies CIE as well as $(*)$ and therefore so does $\pi$ by Lemma 3.5. For the pullback property from $\mathscr{B}(U)$ to $\operatorname{BMOA}(B)$, see the proof of [AR1, Theorem 3.3]. 
Let us look at some consequences of Theorem 3.7.

Boundary behavior. It is known [B] that there exists a Bloch function $g$ on $U$ such that there is no curve in $U$ approaching $T$ along which $g$ has a finite limit. Let $\pi$ be a function as in Theorem 3.7 and define $f=g \circ \pi$. Then by Theorem 3.7 $f \in \operatorname{BMOA}(B)$. It is not hard to see that $f$ satisfies the following:

(a) $f$ has a finite limit along no $\xi$-path for every $\xi \in \operatorname{Max} \pi$, while $f$ extends to a holomorphic function across $S \backslash \operatorname{Max} \pi$. Here, a $\xi$-path is a continuous map $\gamma:[0,1) \rightarrow B$ such that $\gamma(t)$ approaches $\xi$ as $t \rightarrow 1_{-}$.

(b) To each $\xi \in \operatorname{Max} \pi$ corresponds a set $E_{\xi} \subset T$ with $\sigma_{1}\left(E_{\xi}\right)=$ 0 such that $f_{\xi}\left(R_{\eta}\right)$ is dense in $\mathbb{C}$ for every $\eta \in T \backslash E_{\xi}$ where $R_{\eta}$ denotes the radius ending at $\eta$ and $f_{\xi}$ is the slice function $\lambda \rightarrow f(\lambda \xi)$ for $\lambda \in U$.

In fact property (b) is a consequence of Plessner's theorem [G] (see [RR, Theorem 4.2]) and the fact that every slice function of a BMOA-function on $B$ is a Bloch function on $U$ (see for example [AR1]). Note that Max $\pi$ is topologically equivalent to $S^{m_{1}} \times \cdots \times S^{m_{p}} \times T^{m}$ for some $m_{1}, \ldots, m_{p}$ and $m$ such that $p \leq m$ and $1 \leq m_{1}+\cdots+m_{p}+m \leq n$.

Gap Theorem. P. Ahern and W. Rudin [AR2] have recently shown the Paleytype gap theorems for $H^{1}(B)$ (Theorem 3.1 and a part of Theorem 5.3 in [AR2]) in case $\pi \in P_{n}$ is a monomial or the sum-of-squares. The only properties of these $\pi$ (except $\pi \in P_{n}$ ) used in their proof are the following:

(a) $\pi$ has the pullback property from $\mathscr{B}(U)$ to $\operatorname{BMOA}(B)$.

(b) $\left\langle\pi^{m}, \pi^{m}\right\rangle \approx(1 / \sqrt{m})^{\delta(\pi)}$.

The notation $\approx$ means that the ratios of two terms are bounded above and below. The author [C2] has proved that (b) holds for a large class of functions $\pi \in P_{n}$ containing all functions $\pi$ as in Theorem 3.7. We conclude that Ahern-Rudin's Paley-type gap theorems mentioned above hold for every $\pi$ as in Theorem 3.7.

We now give an example which shows that CIE holds only for very special functions $\pi \in P_{n}$. Remark 2.4 in [AR 1] shows that CIE holds only for some special $\pi \in P_{n}: \pi\left(z_{1}, z_{2}\right)=z_{1}^{2}+a z_{2}^{2}(a \neq 0)$ satisfies CIE if and only if $|a|=1$. Also it can be shown that $\pi(z)=a_{1} z_{1}^{2}+\cdots+a_{n} z_{n}^{2} \quad\left(a_{i} \neq 0\right.$ for every i) satisfies CIE if and only if $\left|a_{1}\right|=\cdots=\left|a_{n}\right|=1$. Thus it is tempting to expect that certain "symmetry" plays a significant role in CIE. The following example, however, shows that CIE has nothing to do with "symmetry".

Example 3.8. Assume that $\pi(z)=z_{1}^{d}+\cdots+z_{n}^{d} \in P_{n} \quad(d \geq 2)$ satisfies CIE. We claim that $d=2$. Fix $m \geq 0$, put $\gamma_{m}=\left\langle\pi^{m+1}, \pi^{m+1}\right\rangle /\left\langle\pi^{m}, \pi^{m}\right\rangle$ and let $\alpha=\left(\alpha_{1}, \ldots, \alpha_{n}\right)$ be any multi-index such that $\alpha_{1}+\cdots+\alpha_{n}=m$ and $\alpha_{i} \geq 0$ 
for every $i$. Then, since holomorphic monomials are orthogonal in $L^{2}(\sigma)$, we have by Corollary 3.4

$$
\begin{aligned}
\left\langle\pi^{m+1}, \pi \xi^{d \alpha}\right\rangle & =\gamma_{m}\left\langle\pi^{m}, \xi^{d \alpha}\right\rangle \\
& =\gamma_{m} \frac{\Gamma(m+1)}{\alpha !}\left\langle\xi^{d \alpha}, \xi^{d \alpha}\right\rangle \\
& =\gamma_{m} \frac{\Gamma(m+1) \Gamma(n)}{\Gamma(n+d m)} \frac{(d \alpha) !}{\alpha !}
\end{aligned}
$$

But, by a direct calculation

$$
\begin{aligned}
\left\langle\pi^{m+1}, \pi \xi^{d \alpha}\right\rangle & =\sum_{i=1}^{n}\left\langle\pi^{m+1}, \xi_{i}^{d} \xi^{d \alpha}\right\rangle \\
& =\frac{\Gamma(m+2)}{\alpha !} \sum_{i=1}^{n} \frac{\left\langle\xi_{i}^{d} \xi^{d \alpha}, \xi_{i}^{d} \xi^{d \alpha}\right\rangle}{\left(\alpha_{i}+1\right)} \\
& =\frac{\Gamma(m+2) \Gamma(n)}{\Gamma(n+d m+d)} \frac{(d \alpha) !}{\alpha !} \sum_{i=1}^{n} d\left(d \alpha_{i}+1\right) \cdots\left(d \alpha_{i}+d-1\right)
\end{aligned}
$$

It follows that

$$
m^{1-d} \sum_{i=1}^{n}\left(d \alpha_{i}+1\right) \cdots\left(d \alpha_{i}+d-1\right)=\gamma_{m} \frac{(d m+n) \cdots(d m+d+n-1)}{d m^{d-1}(m+1)}
$$

The right side of the above approaches $d^{d-1}$ as $m \rightarrow \infty$. On the other hand, taking $\alpha_{1}=\cdots=\alpha_{n}=k$ and $m=k n$ in the left side of the above, we obtain

$$
\lim _{k \rightarrow \infty} n^{2-d} k^{1-d}(d k+1) \cdots(d k+d-1)=n^{2-d} d^{d-1}
$$

and therefore $n^{2-d}=1$. In other words $d=2$ (recall that $n \geq 2$ ).

The above example leads to a natural question: is there any concrete characterization of functions $\pi \in P_{n}$ (or $H_{n}$ ) satisfying CIE? We will see a rather abstract characterization at the beginning of $\S 5$.

\section{Some general facts}

The Ahern-Rudin problem is in essence a duality problem in view of the fact that $\mathscr{B}(U)$ is the dual of so-called weighted Bergman spaces (see for example [A]). In this section we obtain some general facts related to the Ahern-Rudin problem in this direction. See Theorem 4.5 and 4.6 below. We will also apply these two theorems to prove Theorem 5.4 in $\S 5$.

Definition 4.1. Suppose $n \geq 1$ and $1 \leq p<\infty$. Let $\pi \in H_{n}$ and $\mu_{\pi}=$ $\sigma\left[\left(\pi^{*}\right)^{-1}\right]$. We let $L^{p}(\pi)=L^{p}\left(\bar{U}, d \mu_{\pi}\right)$ and $A^{p}(\pi)$ denote the closed subspace of $L^{p}(\pi)$ generated by holomorphic polynomials on $\mathbb{C}$. The closed subspace of $H^{p}(B)$ generated by holomorphic polynomials in $\pi$ is denoted by $X^{p}(\pi)$.

The symbol $K_{\pi}$ denotes the orthogonal projection of $L^{2}(\pi)$ onto $A^{2}(\pi)$ and $Q_{\pi}$ the orthogonal projection of $H^{2}(B)$ onto $X^{2}(\pi)$. 
The space $A^{1}(\pi)^{*}$ consists of all $g=K_{\pi}[\psi]$ where $\psi \in L^{\infty}(\pi)=L^{\infty}\left(\bar{U}, d \mu_{\pi}\right)$. The $A^{1}(\pi)^{*}$-norm of $g$ is

$$
\|g\|_{A^{1}(\pi)^{*}}=\inf \left\{\|\psi\|_{L^{\infty}(\pi)}: K_{\pi}[\psi]=g\right\} .
$$

It is immediate from this definition that

$$
\left\|K_{\pi}[\psi]\right\|_{A^{1}(\pi)^{*}} \leq\|\psi\|_{L^{\infty}(\pi)} \text { for } \psi \in L^{\infty}(\pi) .
$$

As before (see $\S 2), A^{1}(\pi)^{*}$ is isometrically isomorphic to the dual of $A^{1}(\pi)$. Clearly $A^{1}(\pi)^{*} \subset A^{2}(\pi)$.

The space $X^{1}(\pi)^{*}$ consists of all $g=Q_{\pi}[\psi]$ where $\psi \in \operatorname{BMOA}(B)$. The $X^{1}(\pi)^{*}$-norm of $g$ is

$$
\|g\|_{X^{1}(\pi)^{*}}=\inf \left\{\|\psi\|_{\mathrm{BMOA}(B)}: Q_{\pi}[\psi]=g\right\} .
$$

Also $X^{1}(\pi)^{*}$ is isometrically isomorphic to the dual of $X^{1}(\pi)$ and $X^{1}(\pi)^{*} \subset$ $X^{2}(\pi)$.

Finally, $D_{\pi}$ is the composition operator $\psi \rightarrow \psi \circ \pi^{*}$ of $L^{2}(\pi)$ into $L^{2}(\sigma)$ and associated with $D_{\pi}$ is the adjoint operator $D_{\pi}^{*}$ of $L^{2}(\sigma)$ into $L^{2}(\pi)$ defined by the requirement that

$$
\int_{S} f \overline{D_{\pi} \psi} d \sigma=\int_{\bar{U}} D_{\pi}^{*} f \bar{\psi} d \mu_{\pi}
$$

for every $f \in L^{2}(\sigma)$ and $\psi \in L^{2}(\pi)$.

Let us look at some examples. Recall that a function $\pi \in H_{n} \quad(n \geq 1)$ is called inner if $\left|\pi^{*}\right|=1[\sigma]$ a.e.

Example 4.2. (1) Let $\pi \in H_{n} \quad(n \geq 1)$ be inner and put $\pi(0)=a$. Then [Ru1] $d \mu_{\pi}(\xi)=P(a, \xi) d \sigma_{1}(\xi)$ where $P$ denotes the Poisson kernel on $U$. It follows that $L^{p}(\pi)$ and $L^{p}\left(\sigma_{1}\right)$ are the same space with two equivalent norms. Moreover, if $\phi_{a}(\lambda)=(a-\lambda) /(1-\bar{a} \lambda) \quad(\lambda \in \bar{U})$, then the map $\psi \rightarrow \psi \circ \phi_{a}$ is a linear isometry of $L^{p}(\pi)$ onto $L^{p}\left(\sigma_{1}\right)$. In particular, if $a=0$, then $L^{p}(\pi)=L^{p}\left(\sigma_{1}\right), A^{p}(\pi)=H^{p}(U), K_{\pi}$ is the Cauchy projection of $L^{2}\left(\sigma_{1}\right)$ onto $H^{2}(U)$ and

$$
Q_{\pi}[f](z)=\int_{S} \frac{f(\xi) d \sigma(\xi)}{1-\pi(z) \bar{\pi}(\xi)} \quad(z \in B)
$$

because $\left\{\pi^{m}\right\}$ is an orthogonal basis for $X^{2}(\pi)$. In general $A^{p}(\pi)$ and $H^{p}(U)$ are the same space with two equivalent norms and hence $L^{p}(\pi) \cap A^{2}(\pi)=A^{p}(\pi)$ for $2 \leq p<\infty$. Note that $A^{1}(\pi)^{*}=\operatorname{BMOA}(U)$ if $a=0$.

(2) Let $\pi \in P_{n}$. It is shown by the author [C2] that $\mu_{\pi} \ll m$ ( $m$ denotes the area measure on $U$ ) and the Radon-Nikodym derivative $w=d \mu_{\pi} / d m$ is a positive $[m]$ a.e., radial, integrable function on $U$. Hence $L^{p}(\pi)=L^{p}(w d m)$. Since $w$ is radial, it is easily seen that $A^{p}(\pi)=L^{p}(\pi) \cap H(U)$ where $H(U)$ is 
the class of functions holomorphic on $U$. In particular, $L^{p}(\pi) \cap A^{2}(\pi)=A^{p}(\pi)$ for $2 \leq p<\infty$. Since $\left\{\pi^{m}\right\}$ is an orthogonal basis for $X^{2}(\pi)$, we have

$$
Q_{\pi}[f](z)=\int_{S} f(\xi) \sum_{m=0}^{\infty} \frac{\pi^{m}(z) \bar{\pi}^{m}(\xi)}{\left\langle\pi^{m}, \pi^{m}\right\rangle} d \sigma(\xi) \quad(z \in B) .
$$

For the convergence of the kernel function see [K].

In what follows $X^{2}(\pi)^{\perp}$ denotes the orthogonal complement of $X^{2}(\pi)$ in $H^{2}(B)$ (not in $L^{2}(\sigma)$ ). We first prove a couple of lemmas which will also be used in the proof of Proposition 5.1 and Theorem 5.3 in $\S 5$.

Lemma 4.3. Suppose $n \geq 1$ and let $\pi \in H_{n}$. Then

$$
\begin{gathered}
D_{\pi}^{*}\left(H^{2}(B)\right)=A^{2}(\pi) \oplus D_{\pi}^{*}\left(X^{2}(\pi)^{\perp}\right), \\
Q_{\pi}=D_{\pi} \circ K_{\pi} \circ D_{\pi}^{*} \text { on } H^{2}(B) .
\end{gathered}
$$

Proof. By a standard approximation we have

$$
\int_{S} \psi \circ \pi^{*} d \sigma=\int_{\bar{U}} \psi d \mu_{\pi} \quad \text { for } \psi \in L^{1}(\pi)
$$

From this we easily find the following:

(a) $D_{\pi}$ is an isometry of $A^{2}(\pi)$ onto $X^{2}(\pi)$,

(b) $D_{\pi}^{*}\left(\pi^{m}\right)=\lambda^{m} \quad(m=0,1, \ldots)$.

It follows that $D_{\pi}^{*}\left(X^{2}(\pi)\right)=A^{2}(\pi)$. On the other hand, given $m \geq 0$ and $h \in X^{2}(\pi)^{\perp}$

and therefore

$$
0=\left\langle\pi^{m}, h\right\rangle=\int_{\bar{U}} \lambda^{m} \overline{D_{\pi}^{*} h} d \mu_{\pi}
$$

$$
D_{\pi}^{*}\left(X^{2}(\pi)^{\perp}\right) \subset A^{2}(\pi)^{\perp}
$$

This proves (1). Note that $D_{\pi} \circ K_{\pi} \circ D_{\pi}^{*}$ annihilates $X^{2}(\pi)^{\perp}$ by (3) and $D_{\pi} \circ$ $K_{\pi} \circ D_{\pi}^{*}$ is the identity on $X^{2}(\pi)$ by (b). This proves (2).

Lemma 4.4. Suppose $n \geq 1$ and $1 \leq p<\infty$. Let $\pi \in H_{n}$. Then for every $h \in L^{2}(\sigma)$

$$
\int_{\bar{U}}\left|D_{\pi}^{*} h\right|^{p} d \mu_{\pi} \leq \int_{S}|h|^{p} d \sigma
$$

holds.

Proof. First consider the case $p=1$. By duality it is sufficient to show that if $\psi \in C(\bar{U})$, then $\left\|\psi \circ \pi^{*}\right\|_{L^{\infty}(\sigma)} \leq\|\psi\|_{L^{\infty}(\pi)}$. Let $E$ be the essential range of $\pi^{*}$. Then $\mu_{\pi}$ lives on $E$. Therefore, if $\psi \in C(\bar{U})$, then

$$
\begin{aligned}
\left\|\psi \circ \pi^{*}\right\|_{L^{\infty}(\sigma)} & \leq \sup \left\{\left|\psi \circ \pi^{*}(\xi)\right|: \xi \in\left(\pi^{*}\right)^{-1}(E)\right\} \\
& \leq \sup \{|\psi(\lambda)|: \lambda \in E\} \\
& =\|\psi\|_{L^{\infty}(\sigma)} .
\end{aligned}
$$


This proves the lemma when $p=1$. A similar duality argument shows the lemma when $1<p<\infty$.

Theorem 4.5. Suppose $n \geq 1$ and let $\pi \in H_{n}$. Then the following are equivalent:

(a) $\left\|C\left[D_{\pi} g\right]\right\|_{\mathrm{BMOA}(B)} \leq c\|g\|_{A^{1}(\pi)^{*}}$ for every $g \in A^{1}(\pi)^{*}$,

(b) $\left\|Q_{\pi}[h]\right\|_{H^{1}(B)} \leq c\|h\|_{H^{1}(B)}$ for every $h \in H^{2}(B)$

where $c=c(n, \pi)$. Two constants in (a) and (b) are the same.

Proof. We first prove the implication (a) $\Rightarrow(\mathrm{b})$. Fix $h \in H^{2}(B)$ and $\psi \in$ $C(\bar{U})$. Then we have

$$
\begin{aligned}
\left|\int_{\bar{U}} K_{\pi}\left[D_{\pi}^{*} h\right] \bar{\psi} d \mu_{\pi}\right| & =\left|\int_{\bar{U}} D_{\pi^{*}}^{*} h \bar{g} d \mu_{\pi}\right| \quad \text { where } g=K_{\pi}[\psi] \\
& =\left|\left\langle h, D_{\pi} g\right\rangle\right| \quad \text { by definition of } D_{\pi}^{*} \\
& \leq\|h\|_{H^{1}(B)}\left\|C\left[D_{\pi} g\right]\right\|_{\mathrm{BMOA}(B)} \quad \text { by definition of } \operatorname{BMOA}(B) \\
& \leq c\|h\|_{H^{1}(B)}\|g\|_{A^{1}(\pi)^{*}} \quad \text { by assumption } \\
& \leq c\|h\|_{H^{1}(B)}\|\psi\|_{L^{\infty}(\pi)} \text { by } 4.1 .(1) .
\end{aligned}
$$

Thus by duality we have

$$
\int_{\bar{U}}\left|K_{\pi}\left[D_{\pi}^{*} h\right]\right| d \mu_{\pi} \leq c\|h\|_{H^{1}(B)} .
$$

But by Lemma 4.3 the left side of the above is exactly the same as $\left\|Q_{\pi}[h]\right\|_{H^{1}(B)}$.

Now we prove the converse implication (b) $\Rightarrow$ (a). Fix $h \in H^{2}(B)$ and $g \in A^{1}(\pi)^{*}$. Then, since $D_{\pi} g \in X^{2}(\pi)$, we have

$$
\left\langle h, D_{\pi} g\right\rangle=\left\langle Q_{\pi}[h], D_{\pi} g\right\rangle=\int_{\bar{U}} D_{\pi}^{*} Q_{\pi}[h] \bar{g} d \mu_{\pi} .
$$

Since $D_{\pi}^{*} Q_{\pi}[h] \in A^{2}(\pi)$, it follows that

$$
\begin{aligned}
\left|\left\langle h, D_{\pi} g\right\rangle\right| & \leq\left\|D_{\pi}^{*} Q_{\pi}[h]\right\|_{A^{1}(\pi)}\|g\|_{A^{1}(\pi)^{*}} \text { by definition of } A^{1}(\pi)^{*} \\
& \leq\left\|Q_{\pi}[h]\right\|_{H^{1}(B)}\|g\|_{A^{1}(\pi)^{*}} \text { by Lemma } 4.4 \\
& \leq c\|h\|_{H^{1}(B)}\|g\|_{A^{1}(\pi)^{*}} \text { by assumption. }
\end{aligned}
$$

In other words, $\left\|C\left[D_{\pi} g\right]\right\|_{\mathrm{BMOA}(B)} \leq c\|g\|_{A^{1}(\pi)^{*}}$. The proof is complete.

We now conclude this section with the following.

Theorem 4.6. Suppose $n \geq 1$ and let $\pi \in H_{n}$. Then $D_{\pi}$ is an isometry of $A^{1}(\pi)^{*}$ onto $X^{1}(\pi)^{*}$.

Proof. Fix $g \in A^{1}(\pi)^{*}$. Given $f \in X^{2}(\pi)$, by Lemma 4.4,

$$
\left|\left\langle f, D_{\pi} g\right\rangle\right| \leq\left\|D_{\pi}^{*} f\right\|_{A^{1}(\pi)}\|g\|_{A^{1}(\pi)^{*}} \leq\|f\|_{H^{1}(B)}\|g\|_{A^{1}(\pi)^{*}}
$$

Thus, by definition of $X^{1}(\pi)^{*}, D_{\pi} g \in X^{1}(\pi)^{*}$ and $\left\|D_{\pi} g\right\|_{X^{1}(\pi)^{*}} \leq\|g\|_{A^{1}(\pi)^{*}}$. 
Conversely, let $h \in X^{1}(\pi)^{*}$. Then $h \in X^{2}(\pi)$ and thus $h=D_{\pi} g$ for some $g \in A^{2}(\pi)$. Now we have for every $f \in A^{2}(\pi)$

$$
\left|\int_{\bar{U}} f \bar{g} d \mu_{\pi}\right|=\left|\left\langle D_{\pi} f, h\right\rangle\right| \leq\left\|C\left[D_{\pi} f\right]\right\|_{H^{1}(B)}\|h\|_{X^{1}(\pi)^{*}}=\|f\|_{A^{1}(\pi)}\|h\|_{X^{1}(\pi)^{*}}
$$

This shows $D_{\pi}$ maps $A^{1}(\pi)^{*}$ onto $X^{1}(\pi)^{*}$ and $\left\|D_{\pi} g\right\|_{X^{1}(\pi)^{*}} \geq\|g\|_{A^{1}(\pi)^{*}}$ for every $g \in A^{1}(\pi)^{*}$. The proof is complete.

\section{Projections}

In this section we observe another consequence of CIE concerning the orthogonal projections $Q_{\pi}$ in Theorem 5.3. As an application of this projection theorem we derive some new information about inner functions which was mentioned at the end of $\S 1$. We begin with a characterization of functions $\pi \in H_{n}$ satisfying CIE in terms of $D_{\pi}^{*}$.

Proposition 5.1. Suppose $n \geq 1$ and let $\pi \in H_{n}$. Then the implications (a) $\Rightarrow$ (b) $\Leftrightarrow(\mathrm{c}) \Leftrightarrow$ (d) hold among the following properties of $\pi$. If $\left\{\pi^{m}\right\}$ is orthogonal in $H^{2}(B)$, then $(\mathrm{b}) \Rightarrow$ (a) also holds.
(a) $\pi$ satisfies $C I E$,
(b) $D_{\pi}^{*}\left(X^{2}(\pi)^{\perp}\right)=0$,
(c) $D_{\pi}^{*}\left(H^{2}(B)\right)=A^{2}(\pi)$,
(d) $D_{\pi}^{*}\left(H^{2}(B)\right) \subset A^{2}(\pi)$.

Proof. By Lemma 4.3 the equivalence of conditions (b), (c) and (d) is easily verified. Now we prove the implication $(\mathrm{a}) \Rightarrow(\mathrm{b})$. Fix $h \in X^{2}(\pi)^{\perp}$ and put $\gamma_{m}=\left\langle\pi^{m+1}, \pi^{m+1}\right\rangle /\left\langle\pi^{m}, \pi^{m}\right\rangle$. By the proof of Theorem 3.6 we have

$$
\left\{\begin{array}{l}
\left\langle\pi^{l}, \pi^{k} h\right\rangle=\gamma_{l-1} \cdots \gamma_{l-k}\left\langle\pi^{l-k}, h\right\rangle=0 \text { for } l \geq k, \\
\left\langle\pi^{l}, \pi^{k} h\right\rangle=\gamma_{l-1} \cdots \gamma_{0}\left\langle 1, \pi^{k-l} h\right\rangle=0 \text { for } l \leq k,
\end{array}\right.
$$

because $h(0)=0$. It follows that

$$
\int_{\bar{U}} \lambda^{l} \bar{\lambda}^{k} \overline{D_{\pi}^{*} h} d \mu_{\pi}=0 \text { for every } l, k \geq 0 .
$$

Since polynomials in $\lambda$ and $\bar{\lambda}$ are dense in $L^{2}(\pi)$, we obtain $D_{\pi}^{*} h=0$ as desired.

Now we suppose that $\left\{\pi^{m}\right\}$ is orthogonal in $H^{2}(B)$ and prove the converse implication $(b) \Rightarrow(a)$. We will show

$$
\left\langle\pi^{m+1}, \pi h\right\rangle=\gamma_{m}\left\langle\pi^{m}, h\right\rangle \text { for every } h \in H^{2}(B) .
$$

Fix $h \in H^{2}(B)$. Since $\left\{\pi^{m}\right\}$ is an orthogonal basis for $X^{2}(\pi)$, we have

$$
Q_{\pi}[h]=\sum_{m=0}^{\infty} \frac{\left\langle h, \pi^{m}\right\rangle}{\left\langle\pi^{m}, \pi^{m}\right\rangle} \pi^{m} .
$$


Therefore, since $D_{\pi}^{*}\left(\pi^{m}\right)=\lambda^{m}$ and $D_{\pi}^{*}\left(X^{2}(\pi)^{\perp}\right)=0$, we see

$$
D_{\pi}^{*} h=D_{\pi}^{*} Q_{\pi}[h]=\sum_{m=0}^{\infty} \frac{\left\langle h, \pi^{m}\right\rangle}{\left\langle\pi^{m}, \pi^{m}\right\rangle} \lambda^{m} .
$$

Note that $\left\{\lambda^{m}\right\}$ is also an orthogonal basis for $A^{2}(\pi)$. Consequently,

$$
\begin{aligned}
\left\langle\pi^{m+1}, \pi h\right\rangle & =\int_{\bar{U}} \lambda^{m+1} \bar{\lambda} \sum_{l=0}^{\infty} \frac{\left\langle\pi^{l}, h\right\rangle}{\left\langle\pi^{l}, \pi^{l}\right\rangle} \bar{\lambda}^{l} d \mu_{\pi} \\
& =\frac{\left\langle\pi^{m}, h\right\rangle}{\left\langle\pi^{m}, \pi^{m}\right\rangle} \int_{\bar{U}}|\lambda|^{2 m+2} d \mu_{\pi} \\
& =\gamma_{m}\left\langle\pi^{m}, h\right\rangle .
\end{aligned}
$$

This shows (1) and the proof is complete.

Remark 5.2. Property (d) above plays an important role in the proof of BMOAresults in [A] and [Rus]. As mentioned earlier, the new proof of these results in [AR1] uses property (a). Note that they are equivalent if $\pi \in P_{n}$.

The following theorem generalizes a projection theorem of $\mathrm{W}$. Rudin (see $\S 1)$. Note that every inner function trivially satisfies CIE.

Theorem 5.3. Let $n \geq 1$ and suppose that $\pi \in H_{n}$ is inner or $\pi \in P_{n}$ satisfies $C I E$. Then $Q_{\pi}$ extends to a bounded projection, with operator norm 1 , of $H^{p}(B)$ onto $X^{p}(\pi)$ for $1 \leq p<\infty$.

Proof. By Proposition $5.1 D_{\pi}^{*}\left(H^{2}(B)\right)=A^{2}(\pi)$. Thus $Q_{\pi}=D_{\pi} \circ D_{\pi}^{*}$ on $H^{2}(B)$ by Lemma 4.3. It follows from Lemma 4.4 that

$$
\left\|Q_{\pi}[h]\right\|_{H^{p}(B)} \leq\|h\|_{H^{p}(B)}
$$

for every $h \in H^{p}(B) \cap H^{2}(B) \quad(1 \leq p<\infty)$. Hence $Q_{\pi}$ is a bounded projection of $H^{p}(B)$ onto $H^{p}(B) \cap X^{2}(\pi)$ for $2 \leq p<\infty$. For $1 \leq p<2, Q_{\pi}$ extends to a bounded projection of $H^{p}(B)$ onto $X^{p}(\pi)$ by (1) because $H^{2}(B)$ and $X^{2}(\pi)$ are dense in $H^{p}(B)$ and $X^{p}(\pi)$, respectively. Clearly the operator norm of $Q_{\pi}$ is 1 in any case.

It remains to prove $H^{p}(B) \cap X^{2}(\pi)=X^{p}(\pi)$ for $2 \leq p<\infty$. Clearly $H^{p}(B) \cap X^{2}(\pi) \supset X^{p}(\pi)$. Suppose $h \in H^{p}(B) \cap X^{2}(\pi)$. Then $h=D_{\pi} g$ for some $g \in A^{2}(\pi)$. In addition $g \in A^{2}(\pi) \cap L^{p}(\pi)=A^{p}(\pi)$ (see Example 4.2). It follows that $h \in X^{p}(\pi)$. The proof is complete.

Theorem 5.4. Let $\pi \in H_{n}(n \geq 1)$ be an inner function such that $\pi(0)=0$. Then the map $g \rightarrow g \circ \pi$ is a linear isometry of $\operatorname{BMOA}(U)$ into $\operatorname{BMOA}(B)$.

Proof. Fix $g \in \operatorname{BMOA}(U)$. Since $\pi(0)=0, A^{1}(\pi)^{*}=\operatorname{BMOA}(U)$. Thus by Theorems 4.5 and 5.3

$$
\left\|C\left[g^{*} \circ \pi^{*}\right]\right\|_{\mathrm{BMOA}(B)} \leq\|g\|_{\mathrm{BMOA}(U)} .
$$


It is known (see for example [Ru1]) that $(g \circ \pi)^{*}=g^{*} \circ \pi^{*}[\sigma]$ a.e. and therefore we have

$$
\|g \circ \pi\|_{\mathrm{BMOA}(B)} \leq\|g\|_{\mathrm{BMOA}(U)} .
$$

Now since $g \circ \pi \in X^{2}(\pi) \cap \operatorname{BMOA}(B)$, we have by definition of $X^{1}(\pi)^{*}$ and Theorem 4.6

$$
\|g\|_{\mathrm{BMOA}(U)}=\|g \circ \pi\|_{X^{1}(\pi)^{*}} \leq\|g \circ \pi\|_{\mathrm{BMOA}(B)} .
$$

The proof is complete.

Corollary 5.5. Let $\pi \in H_{n}(n \geq 1)$ be inner and put $\pi(0)=a$. Then for every $g \in \operatorname{BMOA}(U)$

$$
\|g \circ \pi\|_{\mathrm{BMOA}(B)}=\left\|g \circ \phi_{a}\right\|_{\mathrm{BMOA}(U)}
$$

holds. Here $\phi_{a}(\lambda)=(a-\lambda) /(1-\bar{a} \lambda)(\lambda \in \bar{U})$.

Proof. Apply Theorem 5.4 to the inner function $\phi_{a} \circ \pi$ and use the Möbiusinvariance of $\operatorname{BMOA}(U)$.

Remark 5.6. As a special consequence of Corollary 5.5 we have $g \circ \pi \in$ $\operatorname{BMOA}(B)$ whenever $g \in \operatorname{BMOA}(U)$ and $\pi \in H_{n}(n \geq 1)$ is inner. As far as this property is concerned, $P$. Ahern pointed out more, namely, that the same holds whenever $g \in \operatorname{BMOA}(U)$ and $\pi \in H_{n} \quad(n \geq 1)$. Sketch of proof. It is easy enough to assume $\pi(0)=0$. Let

$$
D f(\lambda)=\int_{S} \frac{f(\xi) d \sigma(\xi)}{1-\lambda \bar{\pi}(\xi)} \quad(\lambda \in U) \text { for } f \in H^{2}(B) .
$$

Then

$$
|D f(\lambda)| \leq 2 \int_{S}|f(\xi)| \operatorname{Re}\left(\frac{1}{1-\lambda \bar{\pi}(\xi)}\right) d \sigma(\xi)+|f(0)|
$$

and therefore $\|D f\|_{H^{1}(U)} \leq 3\|f\|_{H^{1}(B)}$. By duality we have $\|g \circ \pi\|_{\mathrm{BMOA}(B)} \leq$ $3\|g\|_{\mathrm{BMOA}(U)} \square$. Note that this proof depends on the positivity of real part of the Cauchy kernel on $U$, which is not the case when $n \geq 2$. In fact it can be shown that there exists a holomorphic (even continuous up to the boundary) map $\Pi: B_{n} \rightarrow B_{2}$ such that $g \circ \Pi \notin \operatorname{BMOA}\left(B_{n}\right)$ for some $g \in \operatorname{BMOA}\left(B_{2}\right)$.

\section{REFERENCES}

[A] P. Ahern, On the behavior near torus of functions holomorphic in the ball, Pacific J. Math. 107 (1983), 267-278.

[ACP] J. M. Anderson, J. Clunie and Ch. Pommerenke, On Bloch functions and normal functions, J. Reine Angew. Math. 270 (1974), 12-37.

[AR1] P. Ahern and W. Rudin, Bloch functions, BMO and boundary zeros, Indiana Math. J. 36 (1987), 131-148.

[AR2] _, Paley-type gap theorems for $H^{p}$-functions on the ball, Indiana Univ. Math. J. (to appear).

[B] K. G. Binmore, Analytic functions with Hadamard gaps, Bull. London Math. Soc. 1 (1969), 211-217.

[C1] B. R. Choe, Composition with bounded holomorphic functions on the ball, Ph.D. thesis, Univ. of Wisconsin-Madison, 1988. 
[C2] _ - Weights induced by homogeneous polynomials, Pacific J. Math. (to appear).

[CRW] R. Coifman, R. Rochberg and G. Weiss, Factorization theorems for Hardy spaces in several variables, Ann. of Math. 103 (1976), 611-635.

[G] J. B. Garnett, Bounded analytic functions, Academic, New York, 1981.

[K] C. Kolaski, Projections onto spaces of holomorphic functions, Indiana Univ. Math. J. 29 (1980), 769-775.

[RR] W. Ramey and P. Russo, Behavior of functions in BMOA and VMOA near (2n-2)-dimensional submanifold, Indiana Math. J. 37 (1988), 73-81.

[Ru1] W. Rudin, Composition with inner functions, Complex Variables 4 (1984), 7-19.

[Ru2] _ Function theory in the unit ball of $\mathbb{C}^{n}$, Springer-Verlag, Berlin, Heidelberg and New York, 1980.

[Rus] P. Russo, Boundary behavior of $\operatorname{BMO}\left(B_{n}\right)$, Trans. Amer. Math. Soc. 292 (1985), 733-740.

[U] D. Ullrich, Radial divergence in BMOA, preprint.

Department of Mathematics, University of Wisconsin, Madison, Wisconsin 53706

Current address: Department of Mathematics, Michigan State University, East Lansing, Michigan 48824 